\title{
De Novo Design of Allosteric Control into Rotary Motor V1-ATPase
}

\author{
by Restoring Lost Function
}

${ }^{1}$ Research Center of Integrative Molecular Systems, Institute for Molecular Science (IMS), National 7 Institutes of National Sciences (NINS), Okazaki, Aichi, 444-8585, Japan

$8 \quad{ }^{2}$ Exploratory Research Center on Life and Living Systems (ExCELLS), National Institutes of

9 National Sciences, Okazaki, Aichi, 444-8585, Japan

$10 \quad{ }^{3}$ Department of Structural Molecular Science, School of Physical Sciences, SOKENDAI (The

11 Graduate University for Advanced Studies), Hayama, Kanagawa, 240-0193, Japan

$12{ }^{4}$ Department of Life and Coordination-Complex Molecular Science, Institute for Molecular Science (IMS), National Institutes of National Sciences (NINS), Okazaki, Aichi, 444-8585, Japan

${ }^{5}$ Department of Functional Molecular Science, School of Physical Sciences, SOKENDAI (The

Graduate University for Advanced Studies), Hayama, Kanagawa, 240-0193, Japan

${ }^{6}$ Structural Biology Research Center, Institute of Materials Structure Science, High Energy

Accelerator Research Organization (KEK), Tsukuba, Ibaraki, 305-0801, Japan

* To whom correspondence should be

addressed. Tel: 81-564-55-7379. E-mail: 


\section{${ }_{21}$ Abstract}

Protein complexes exert various functions through allosterically controlled cooperative work. De novo design of allosteric control into protein complexes provides understanding of their working principles and potential tools for synthetic biology. Here, we hypothesized that an allosteric control can be created by restoring lost functions of pseudo-enzymes contained as subunits in protein complexes. This was demonstrated by computationally de novo designing ATP binding ability of the pseudo-enzyme subunits in a rotary molecular motor, $\mathrm{V}_{1}$-ATPase. Single molecule experiments with solved crystal structures revealed that the designed $\mathrm{V}_{1}$ is allosterically accelerated than the wild-type by the ATP binding to the created allosteric site and the rate is tunable by modulating the binding affinity. This work opened up an avenue for programming allosteric control into proteins exhibiting concerted functions. 
Protein complexes exert their various functions through the cooperative work between their constituent subunits ${ }^{1,2}$. The orchestration between the subunits is enabled by the allosteric mechanism, in which a protein function in an active site is controlled by the response to stimuli that occurs at a site away from the active site ${ }^{3}$. The design of allosteric control into protein complexes to reveal their working principles and provide novel functionalities has been attempted ${ }^{4-9}$. One of these approaches is to create fusion proteins between the target protein, whose functions are to be controlled, and a protein undergoing conformational changes in response to stimuli, such as the binding of an effector molecule $^{6,7}$ or light absorption ${ }^{8,9}$. Nakamura et al. created the remotely controlled linear motor protein with a light-sensitive domain ${ }^{8}$. In this study, we sought an approach to program allosteric control into protein complexes by creating binding sites for an allosteric effector molecule in the complexes.

We focused on pseudo-enzymes, which are homologs of some enzymes but are proven or predicted to have lost their enzymatic activity ${ }^{10-12}$. The overall structures of pseudo-enzymes are similar to those of the enzymes, but the conserved amino acids required for their functions are lost from the active sites; therefore, these sites are called pseudo-active sites. Interestingly, it has been reported that such pseudo-enzymes exhibit allosteric control when they form complexes ${ }^{10,11}$. For example, a complexforming pseudo-kinase — the pseudo-active site can bind ATP but has lost kinase activity—activates the catalytic function of a complex-forming partner protein, by ATP binding at the pseudo-active 
site $^{13,14}$. Here, we hypothesized that an allostery can be de novo designed into protein complexes by restoring the lost function of pseudo-enzymes included in the complexes (Fig. 1a).

A pseudo-enzyme is found in a protein complex, the rotary motor $V_{1}$-ATPase $\left(V_{1}\right) . V_{1}$ is a part of an ion pump V-ATPase which transports cations across the membrane by ATP hydrolysis-driven rotation ${ }^{15}$. The $\mathrm{V}_{1}$ consists of a rotor composed of the D- and F- subunits and the stator of the hexametric ring composed of three A-subunits and three B-subunits (Fig. 1b) ${ }^{16}$. The B-subunit is a pseudo-enzyme of the A-subunit and has homology with the A-subunit (e.g. for Enterococcus hirae $\mathrm{V}_{1}$-ATPase, the BLAST E-value between the subunits is $4 \times 10^{-22}$ ) and the overall subunit structures resemble each other (Supplementary Fig. 1). However, the details of their sequences and structures are different, resulting in the two different interfaces between the A- and $\mathrm{B}$ - subunits in the $\mathrm{A}_{3} \mathrm{~B}_{3}$ hexameric ring. One is the catalytic interface, at which the A-subunit has an ATP hydrolysis catalytic site, and the other is the non-catalytic interface, at which the B-subunit has a pseudo-active site, which does not hydrolyze or even bind $\mathrm{ATP}^{16,17}$ (Supplementary Fig. 2).

The relation of the pseudo-active site to the activity at the active site is reported for $\mathrm{V}_{1}$, in which the mutations in the pseudo-active site decrease its activity ${ }^{18,19}$. Furthermore, a study on the rotary motor $\mathrm{F}_{1}$-ATPase from the thermophilic Bacillus PS3, which shares a common ancestor with $\mathrm{V}_{1}$-ATPase ${ }^{20}$, reported the relationship between the pseudo-active site and the active site in the rotational 
mechanism ${ }^{21}$. Similar to $V_{1}, F_{1}$ has the stator $\alpha_{3} \beta_{3}$ ring complex, in which the $\beta$-subunit has ATP hydrolase ability, while the $\alpha$-subunit, a pseudo-enzyme subunit of the $\beta$-subunit, can bind but not hydrolyze $\mathrm{ATP}^{20,22}$. The mutation in the pseudo-active site in the $\alpha$-subunit, which significantly decrease the ATP binding ability, was found to cause $F_{1}$ to have long pauses more frequently than the wild-type. This is likely because of the impeded release of ADP at the active site, indicating that the pseudo-active site away from the active site allosterically impacts on the active site's function ${ }^{21}$. This study leads to the hypothesis that the pseudo-active site in the non-catalytic interface of $\mathrm{V}_{1}$, which does not have ATP binding ability ${ }^{16,17}$, can be a target for de novo design of a binding site for an allosteric effector molecule. We tested this hypothesis by computationally designing an ATP-binding site at the pseudo-active site in Enterococcus hirae $\mathrm{V}_{1}$-ATPase. 


\section{Results}

\section{Computational design of an allosteric site in the pseudo-active site}

The pseudo-active site in the B-subunit does not have space for nucleotide binding or the well-known loop motif for phosphate binding, the Walker-A motif $\left(\mathrm{GX}_{1} \mathrm{X}_{2} \mathrm{X}_{3} \mathrm{X}_{4} \mathrm{GK}[\mathrm{T} / \mathrm{S}]\right)^{23,24}$, also called a P-loop (Supplementary Fig. 2). Recently, computational methods for designing small molecule binding proteins have been developed, using Rosetta design software ${ }^{25-27}$. We attempted to computationally design an ATP binding site de novo together with a P-loop at the pseudo-active site in the B-subunit monomer, using Rosetta with a set of features for P-loops obtained from statistical analyses of naturally occurring proteins (Supplementary Fig. 3).

First, the backbone structure of the P-loop was built at the pseudo-active site by using the backbone of the A-subunit's P-loop, considering the P-loop orientation feature (Supplementary Fig. 3a). Subsequently, side-chain conformations (amino acid sequences) of the P-loop and the surrounding residues, which have favorable interactions with ATP, are explored with various ATP conformations, the feature for native P-loops for the conserved amino acid (Gly) at $\mathrm{X}_{3}$ (Supplementary Fig. 3c), and the typical distances between the atoms of the P-loop and the phosphate atoms of ATP (Supplementary

Fig. 3d). The resulting designed structures bound to an ATP were energetically minimized. This sequence design followed by energy minimization was iterated, and the designs with high ATP binding 
ability predicted by the Rosetta score were selected; the designs that lost the feature for the conserved 


\section{Designed B-subunits forms a ring complex with A-subunits}

The designed B-subunit (De), expressed with the A-subunit in E. coli using the plasmid pTR19-

101

$\mathrm{AB}^{28}$ and purified by a $\mathrm{Ni}^{2+}$-affinity chromatography followed by size exclusion chromatography, formed a ring complex with the A-subunit $\left(\mathrm{A}_{3}(\mathrm{De})_{3}\right.$ ring complex) (Supplementary Fig. 7a). Subsequently, to evaluate the ATP binding ability of De, we introduced a double mutation in the Asubunit (K238A and T239A) to significantly impair ATP binding ability ${ }^{29}$. However, De did not form the $\mathrm{A}_{3}(\mathrm{De})_{3}$ ring complex with the mutant A-subunit (Supplementary Fig. 7b). Therefore, we purified De as a monomer (Supplementary Fig. 7c) and the nucleotide binding ability was indirectly evaluated by thermal shift ${ }^{30}$ in circular dichroism spectroscopy in the presence or absence of nucleotides (Supplementary Fig. 8). The De monomer exhibited an increase of its melting temperature upon the addition of nucleotide, while the melting temperatures for the wild-type B-subunit monomer was almost the same in the presence and absence of nucleotides, suggesting that De has nucleotide binding ability. Thus, we attempted to determine the crystal structures of the $A_{3}(\mathrm{De})_{3}$ complex to prove the nucleotide-binding ability of De. 


\section{The designed B-subunit binds to nucleotide}

First, the $\mathrm{A}_{3}(\mathrm{De})_{3}$ complex was crystallized in the absence of nucleotide and the structure was solved

at $2.77 \AA$ resolution, named $\mathrm{A}_{3}(\mathrm{De})_{3} \_$empty. This showed the hexameric ring structure without

nucleotide, which is the same as the wild-type structure (Fig. 2a). The structure of the designed site in

the B-subunit in the crystal structure was almost identical to the computationally designed model

(Supplementary Fig. 9a,b). When we incubated the nucleotide-free crystals with $20 \mu \mathrm{M}$ AMP-PNP for

5 hours, we found an extra density corresponding to AMP-PNP in a catalytic site. The structure,

$\mathrm{A}_{3}(\mathrm{De})_{3} \_(\mathrm{ANP})_{1 \text { cat, }}$ was solved at $3.44 \AA$ resolution (Fig. 2b). Next, the nucleotide-free crystals were

incubated for 5 hours with ADP by gradually increasing ADP concentration to $10 \mathrm{mM}$ and the resulting

structure, $\quad \mathrm{A}_{3}(\mathrm{De})_{3} \_(\mathrm{ADP} \cdot \mathrm{Pi})_{1 \text { cat }}(\mathrm{ADP})_{2 \text { cat,2non-cat, }}$

was solved at

2.90

$\AA$ resolution. In

$\mathrm{A}_{3}(\mathrm{De})_{3}(\mathrm{ADP} \cdot \mathrm{Pi})_{1 \text { cat }}(\mathrm{ADP})_{2 \text { cat,2non-cat, }}$ each of the three catalytic sites is occupied by ADP (one of the

sites has ADP with a possible Pi) and each of the two sites out of the three design sites is with ADP

(Fig. 2c). This crystal structure proved that the designed site in the non-catalytic interface has the

Fig. 9c,d). Furthermore, the nucleotide-free crystals were incubated overnight by gradually 
130 sites and one of the designed sites are occupied by ADP (Fig. 2d), and the other is

$131 \mathrm{~A}_{3}(\mathrm{De})_{3}(\mathrm{ADP})_{3 \text { cat,2non-cat, }}$ in which each of the three catalytic sites and each of the two designed sites are occupied by ADP (Fig. 2e). Although the resolution of these structures is relatively low (3.95 $\AA$ ), the densities for the main chain C $\alpha$-trace and bound ADPs were clearly observed. The nucleotide-

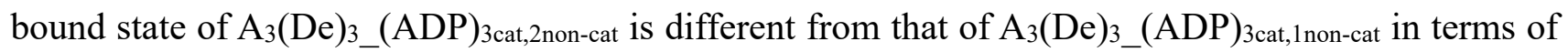
nucleotide occupation at the designed site; these structures may provide the allosteric response upon

ADP binding in the designed site (details are described later and in Fig. 5d). 


\section{Creation of ATP binding sites at the non-catalytic interfaces impacts on the}

\section{catalytic interfaces} and ADP (Mant-ADP), revealed that the nucleotide-binding affinities of the designed complex are much lower than those of the wild-type complex (Fig. 3a). The measured binding affinity of the $\mathrm{A}_{3} \mathrm{~B}_{3}$ and $\mathrm{A}_{3}(\mathrm{De})_{3}$ complexes respectively were $14.5 \pm 5.7 \mathrm{nM}$ and $2.03 \pm 0.18 \mu \mathrm{M}$ for AMP-PNP, and 64.2 $\pm 0.9 \mathrm{nM}$ and $0.55 \pm 0.003 \mu \mathrm{M}$ for ADP. Note that the affinities of $\mathrm{A}_{3}(\mathrm{De})_{3}$, measured in a range of relatively low nucleotide concentration, are expected for the first nucleotide binding to one of the catalytic interfaces, as supposed by the $\mathrm{A}_{3}(\mathrm{De})_{3}(\mathrm{ANP})_{1 \text { cat }}$ structure, in which a single nucleotide was bound to one of the catalytic interfaces (Fig. 2b). Structure comparison of $A_{3}(\text { De })_{3}$ empty with the nucleotide-free wild-type $\mathrm{A}_{3} \mathrm{~B}_{3}$ provides an interpretation for the decreased affinities (Fig. 3b). The Ploops in the three A-subunits in the wild-type $\mathrm{A}_{3} \mathrm{~B}_{3}$ complex are classified into the two distinct conformations, bound and unbound forms (Supplementary Fig. 10). The P-loop in an A-subunit is the bound form and those in the other two A-subunits are the unbound form (Fig. 3b, top). The bound 
lower affinities of the catalytic interface. In other words, the creation of ATP binding sites in the noncatalytic interfaces changed the conformations of the ATP binding sites in the catalytic interfaces. 


\section{Creation of ATP binding sites induces global conformational change of the $A_{3}(\mathrm{De})_{3}$}

\section{complex}

The comparison of the $\mathrm{A}_{3}(\mathrm{De})_{3}$ _empty with the nucleotide-free wild-type complex revealed that conformational changes occurred not only in the P-loop of the A-subunits but also in the overall structure of the A-subunits (Fig. 3b, bottom), although remarkable conformational changes were not found in any of the B-subunits (the structures of $\alpha$-helical domains are locally different from the wildtype B-subunits due to their innate flexibility) (Fig. 3d). In the nucleotide-free wild-type complex, the A-subunit, of which the P-loop is the bound form, shows the closed conformation (the C-terminal domain bends toward the pore of the ring complex), and the other two A-subunits with the unbound form are in the open conformation ${ }^{16}$. However, all A-subunits in the design complex, $\mathrm{A}_{3}(\mathrm{De})_{3}$ empty, show the open conformation with the unbound form (Fig. 3b). Furthermore, these conformational changes broke the asymmetric arrangement of A- and B- subunits found in the wild-type ring complex ${ }^{16}$, resulting in a nearly symmetric arrangement with the expansion of the ring pore in the design complex (Fig. 3c). This observation leads to the hypothesis that the central rotor is difficult to retain in the expanded pore. As expected, the reconstitution experiments of the rotor (D- and Fsubunits) and ring (A- and B- subunits) complex show that the reconstitution ratio was quite low (Supplementary Fig. 11). Interestingly, the conformational changes observed in $\mathrm{A}_{3}(\mathrm{De})_{3}$ empty were 
173 (Supplementary Fig. 11), and the complex reconstitution ratio was also recovered in the presence of

174 nucleotide (Supplementary Fig. 12). The detailed comparisons of $\mathrm{A}_{3}(\mathrm{De})_{3_{3} \text { empty and }}$

$175 \mathrm{~A}_{3}(\mathrm{De})_{3}{ }_{-}(\mathrm{ANP})_{1 \text { cat }}$ with the nucleotide-free wild-type complex structure ${ }^{16}$ are described in

176 Supplementary Tables $1-4$. 


\section{The designed $V_{1}$ rotates faster than the wild-type}

Finally, we carried out single-molecule experiments to observe the rotation of the $A_{3}(\mathrm{De})_{3} \mathrm{DF}$ complex in various ATP concentrations ([ATP $]$ s). The designed $\mathrm{V}_{1}$ was found to rotate unidirectionally in a counterclockwise fashion with discrete $120^{\circ}$ steps, similar to the wild-type, but rotates faster than the wild-type at $100 \mu \mathrm{M}$ ATP (Fig. 4a). Furthermore, in contrast to the wild-type, the rotation rate of the designed $\mathrm{V}_{1}$ exhibited an unique non-Michaelis-Menten type dependence on [ATP]. Rotation rates very similar to the wild-type were observed at the lowest $(1 \mu \mathrm{M})$ and the highest $(30 \mathrm{mM})[\mathrm{ATP}]$, but compared with the wild-type, can be tuned by modulating the nucleotide-binding affinity of the designed site (Fig 4b). The mutant K157Q at one of the conserved residues in P-loop motif in the 
was observed, shifted higher from that for the original design: $1 \mathrm{mM}$ [ATP](Fig. $4 \mathrm{~b}$, middle). In

addition, the double mutant K157A/S158A in the P-loop, which has a further decreased binding

concentration was the highest $(161 \pm 18 \mathrm{rps})$ among those for the wild-type, the original design and

200 the mutants (Fig. 4b). This observed correlation between the nucleotide binding affinity of the designed

201 site and the $[\mathrm{ATP}]$ at which the most acceleration is observed strongly suggests the allosteric effect

202 produced by the nucleotide-binding to the designed site. 


\section{ADP-release at the catalytic site is facilitated allosterically}

In the $120^{\circ}$ step rotation, the designed $\mathrm{V}_{1}$ was found to have the main-pauses and sub-pauses before

the $40^{\circ}$ and $80^{\circ}$ sub-steps respectively, which is the same as the wild-type $\mathrm{V}_{1}{ }^{28}$ (Fig. 5a). To reveal the

mechanism of allosteric acceleration, we carried out dwell-time analyses of the two pauses at the high

and low [ATP] $\left(1 \mu \mathrm{M}\right.$ and $30 \mathrm{mM}$, respectively), in which the designed $\mathrm{V}_{1}$ rotated at a similar rate as

the wild-type, and at the $100 \mu \mathrm{M}$ [ATP], in which the design exhibited the most accelerated rotation.

In the proposed rotation model for the wild-type ${ }^{28}$, the main-pause corresponds to the dwell-time waiting for ATP-binding, ATP-hydrolysis, and Pi-release, and the sub-pause corresponds to that for

ADP-release. The main-pause time constants of the design at each measured [ATP] are roughly the

same as those of the wild-type, irrespective of [ATP] (Fig. 5b). The sub-pause time constants for the

wild-type stayed constant between $2.1 \sim 2.7 \mathrm{~ms}$, at any [ATP]s, and the time constants for the design at

the low and high [ATP] are similar to those for the wild-type. However, the time constant at the [ATP]

(100 $\mu \mathrm{M}$ ATP), at which the designed $\mathrm{V}_{1}$ showed the most accelerated rate, significantly decreased to 
origin of the acceleration is the facilitated ADP-release at the catalytic sites, which is generated through

221 the allosteric effect triggered by nucleotides binding to the designed sites.

222 Structural comparisons between the solved structures, $\mathrm{A}_{3}(\mathrm{De})_{3}(\mathrm{ADP})_{3 \text { cat, } 1 \text { non-cat }}$ and

$223 \mathrm{~A}_{3}(\mathrm{De})_{3}(\mathrm{ADP})_{3 \mathrm{cat}, 2 \text { non-cat, }}$ provide a structure-based interpretation for ADP-release promoted by the

224 allosteric effect, although these structures are bound not with ATP but ADP, and do not contain the

225 rotor. An ATP-binding at the designed site is suggested to induce conformational changes of the

226 neighboring A-subunit and the catalytic interface from the closed conformation to the open-like

227 conformation (Fig. 5c,d and Supplementary Tables 7 and 8), which creates space to facilitate ADP

228 release. 


\section{Possible model of the rotation scheme for designed $V_{1}$} an empty catalytic site triggers a $40^{\circ}$ sub-step, and the subsequent release of ADP from the neighbor catalytic-site generates the $80^{\circ}$ sub-step. The design may rotate in a similar scheme except for the ATPbinding to one or two designed sites at the non-catalytic interfaces, which facilitates ADP-release in the neighboring catalytic site through an allosteric effect (Fig. 5e, right). It is obvious that the allosteric effect does not emerge at low [ATP] since the designed sites are not able to bind ATP due to low affinity, but it is still puzzling why the allosteric effect is not observed in high [ATP]; the full nucleotide occupation in the three designed sites may suppress the allosteric effect, but, this should be verified in the future. 


\section{Implications to native $V_{1}$-ATPase and a common mechanism for rotary motors}

The designed $\mathrm{V}_{1}$ not only exhibited the allosteric control over the rotation, but also provided possible designs and working principles for the native $\mathrm{V}_{1}$-ATPase. It is suggested that ancestral $\mathrm{V}_{1}$-ATPase existed as a homo hexameric ring, in which all subunits perform ATP binding and hydrolase functions, which has since evolved to form the current hetero hexameric ring containing the pseudo-enzyme subunit (B-subunit) that lost these functions ${ }^{20}$. Restoring ATP binding ability at the pseudo-active site may lead to an understanding of why the modern, descendant $\mathrm{V}_{1}$-ATPase lost its ATP binding and hydrolase functions. First, it is plausible that the non-catalytic interface observed in the modern $\mathrm{V}_{1^{-}}$ ATPase plays a role in attaining the Michaelis-Menten type rotation, in which the rotation rate is smoothly regulated along an [ATP] (Fig. 4b). The sudden increase and decrease of rotation rate at an [ATP] as observed in the designed $\mathrm{V}_{1}$ would not be preferable in terms of functional regulation by nature (this property can be beneficial for human since $\mathrm{V}_{1}$ can be engineered with the maximal rotation rate at an arbitrary $[\mathrm{ATP}])$. Second, the non-catalytic interface may be a key factor for making the asymmetrical ring shape observed in the modern $\mathrm{V}_{1}$-ATPase in the absence of nucleotides, as we observed that the designed $\mathrm{V}_{1}$ forms a nearly symmetric ring structure (Fig. 3b,c). The asymmetrical ring shape is considered to be one of the key factors to realize the unidirectional rotation ${ }^{16}$ (it is reported that the $\mathrm{N}$-terminal $\beta$ barrel domains in the $\mathrm{A}$ - and $\mathrm{B}$-subunits also play this role ${ }^{32}$ ). At the end, restoring 
ATP binding ability also implies a common mechanism for hexameric rotary motors of $\mathrm{V}_{1}$ and $\mathrm{F}_{1}$ : the 


\section{Conclusion}

264 We succeeded in programing allosteric control into the molecular motor $\mathrm{V}_{1}$-ATPase by computational

de novo design of a ATP binding site at the pseudo-active site in the non-catalytic interface of $\mathrm{V}_{1}$.

266 Furthermore, the artificially designed $\mathrm{V}_{1}$ provided implications for design and working principles of

267 the native $\mathrm{V}_{1}$-ATPase. Pseudo-enzymes are frequently found in native complex-forming proteins, e.g.

$268 \mathrm{~F}_{1}$-ATPase ${ }^{22}$, dynein $^{33}$, kinesin $^{34}, 20 \mathrm{~S} \mathrm{proteasome}^{35}$, kinases $^{13}$ and plant resistosome ${ }^{36}$. Engineering

269 pseudo-active sites could be one of the promising approaches for de novo design of allosteric control

270 into complex-forming proteins. 


\section{Methods}

\section{Computational design protocol}

The B-subunit was computationally redesigned using the structure, chain E in PDB 3VR6. First, the

pseudo P-loop in B-subunit (the residues 151-158) were replaced by the P-loop motif of A-subunit (the residues 232-239), by superimposing the A-subunit (chain B in the same PDB 3VR6) to the B-subunit with the orientation feature of P-loop shown in Supplementary Fig. 3a. Second, ATP binding modes were designed using Rosetta design software ${ }^{37}$ with Talaris2014 score function (Parameters for ATP$\mathrm{Mg}^{2+}$ were determined using those for atom types already defined in Rosetta). In the design calculation, side-chain conformations for the residue positions of the P-loop and the surrounding residues (E169, T248, Q339, and F417), having favorable interactions with ATP, were explored with various ATP conformations generated by BCL software ${ }^{38}$ and with the distance constraints between the atoms of Ploop and the phosphate atoms of ATP (Supplementary Fig. 3d) (the amino acid at $\mathrm{X}_{3}$ in P-loop was fixed to Gly (Supplementary Fig. 3c)). Third, the designed B-subunit structures with ATP were minimized. The second and third steps were iterated 20 times and 800 different ATP binding modes were designed. Fourth, the designed structures that lost the feature for conserved backbone torsion pattern of P-loop (Supplementary Fig. 3b) were abandoned, and then those of which ATP binding score 


\section{Molecular dynamics simulations}

The 29 designs obtained by the computational design using Rosetta was further evaluated for their

ATP binding ability by observing the stability of ATP in the designed site during short molecular dynamics (MD) simulations. The AMBER14 software suite ${ }^{39}$ was used for all MD simulations. The

design models were used as the initial structures, of which hydrogen atoms were added by the LEaP

module of AMBER14 ${ }^{39}$. The simulation system contains a designed B-subunit monomer with ATP

placed in a water box of approximately $82 \AA \times 112 \AA \times 100 \AA$. To neutralize the system, $15-17$ sodium

ions were put in the box. AMBER ff99SB sets and TIP3P were utilized for the protein and water

molecules, respectively. Parameters for ATP molecule were adopted from a reference paper ${ }^{40}$. Long

range electrostatic interactions were treated by the particle mesh Ewald (PME) method. Non-bonded

interactions were cut off at $10 \AA$. After carrying out a short minimization to remove artificial repulsions in the initial structure, $10 \mathrm{~ns}$ MD simulations in a constant-NPT $(300 \mathrm{~K}$, 1atm) ensemble were performed after the 100 ps heating stage with NVT ensemble (the time step is 2.0 fs and hydrogen atoms were constrained with SHAKE procedure). At the heating step, the temperature was raised gradually from $0 \mathrm{~K}$ to $300 \mathrm{~K}$ with the weak restraints $\left(10 \mathrm{kcal} / \mathrm{mol} / \mathrm{A}^{2}\right)$ to the atoms of designed $\mathrm{B}$ subunit. The MD simulation trajectory for each designed structure are shown in Supplementary Fig. 6 
minimized structure. Finally, a designed structure showing low RMSD value throughout the MD simulation was selected for experimental characterization. 


\section{Expression and purification of the $\mathrm{A}_{3}(\mathrm{De})_{3}$ Complex}

A DNA fragment of the design was synthesized from the $n t p B$ gene in $\mathrm{pTR} 19-\mathrm{AB}^{28}$ using megaprimer

PCR method, and then the $n t p B$ gene was replaced by this design fragment. The DNA sequence of and $2 \mathrm{mM}$ isopropyl $\beta$-D-thiogalactopyranoside. Grown cells were spun down at 6,000 rpm for 10 minutes and washed twice with buffer $\mathrm{A}\left(20 \mathrm{mM}\right.$ potassium $\mathrm{P}_{\mathrm{i}}(\mathrm{pH} 7.0)$ and $\left.100 \mathrm{mM} \mathrm{NaCl}\right)$. The cells were suspended in $15 \mathrm{~mL}$ of buffer A supplemented with $75 \mu \mathrm{L} 100 \mathrm{mM}$ phenylmethylsulfonyl fluoride (PMSF) solution and subsequently disrupted by sonication. After removing cell debris by centrifugation at $10,000 \mathrm{rpm}$ for 20 minutes at $4{ }^{\circ} \mathrm{C}$, the solution was filtered and applied to a Ni-NTA column. After washing with buffer $\mathrm{B}\left(20 \mathrm{mM}\right.$ potassium $\mathrm{P}_{\mathrm{i}}(\mathrm{pH} 7.0), 230 \mathrm{mM} \mathrm{NaCl}$ and $20 \mathrm{mM}$ Imidazole), $\mathrm{A}_{3}(\mathrm{De})_{3}$ complex was eluted with buffer $\mathrm{C}\left(20 \mathrm{mM}\right.$ potassium $\mathrm{P}_{\mathrm{i}}(\mathrm{pH} 7.0), 50 \mathrm{mM} \mathrm{NaCl}$ 
bioRxiv preprint doi: https://doi.org/10.1101/2020.09.09.288571; this version posted September 9, 2020. The copyright holder for this preprint (which was not certified by peer review) is the author/funder. All rights reserved. No reuse allowed without permission. 


\section{Expression and purification of the design monomer}

The designed B-subunit monomer was obtained by breaking the $A_{3}(\mathrm{De})_{3}$ complex sample in the presence of high concentration ATP. After expression and Ni-NTA purification of the $\mathrm{A}_{3}(\mathrm{De})_{3}$ complex sample by the above described methods, the buffer of the $\mathrm{A}_{3}(\mathrm{De})_{3}$ sample solution eluted from a NiNTA column was exchanged to buffer E (20 mM MES-NaOH (pH 6.5), 10\% Glycerol, $100 \mathrm{mM} \mathrm{KCl}$ and $5 \mathrm{mM} \mathrm{MgSO}_{4}$ ) using PD-10 column (GE Helthcare). The sample solution mixed with $2 \mathrm{mM}$ ATP was rocked for $30-40$ minutes at $4{ }^{\circ} \mathrm{C}$, filtered and applied to a Ni-NTA column. Because A-subunit has a His-tag and designed B-subunit does not, designed B-subunit can be selectively recovered in the flow through. In the flow through sample, Tris-HCl was added (100 mM final concentration; $\mathrm{pH} 8.5)$. The buffer of sample solution was exchanged to buffer F (20 mM Tris-HCl (pH 8.5), 10\% Glycerol, $100 \mathrm{mM} \mathrm{KCl}$ and $5 \mathrm{mM} \mathrm{MgSO}_{4}$ ) by concentrating with a Vivaspin20 5,000 MWCO (Sartorius) and adding buffer F. The samples were passed through a Superdex 200 Increase column (GE Helthcare) equilibrated with buffer F. The purity of design monomer sample was confirmed by SDS-PAGE (Supplementary Fig. 7c). 


\section{Expression and purification of the wild-type B-subunit}

(pH8.5)) supplemented with $125 \mu \mathrm{L}$ of $100 \mathrm{mM}$ PMSF solution, and then disrupted by sonication.

After removing cell debris by centrifugation at 10,000 rpm for 20 minutes at $4{ }^{\circ} \mathrm{C}$, the solution was

Glycerol, $0.7 \mathrm{M} \mathrm{KCl}, 5 \mathrm{mM} \mathrm{MgSO}$, $0.1 \mathrm{mM}$ DTT and $20 \mathrm{mM}$ Imidazole (pH8.5)), B-subunit monomer was eluted with buffer I (20 mM Tris- $\mathrm{HCl}$ (pH 8.5), 5\% Glycerol, $0.7 \mathrm{M} \mathrm{KCl,} 5 \mathrm{mM} \mathrm{MgSO}_{4}$, 


\section{Expression and purification of the DF-subcomplex}

The DF-subcomplex of Enterococcus hirae $\mathrm{V}_{1}$ were expressed in E. coli. BL21* (DE3) competent cells using the pTR19-D(M1G/T60C/R131C)F plasmid ${ }^{28}$. The transformed cells were cultured in Super Broth containing $100 \mu \mathrm{g} / \mathrm{ml}$ ampicillin at $37^{\circ} \mathrm{C}$ for $4-5$ hours until $\mathrm{OD}_{600}$ reached 0.5 , then the temperature was decreased to $30^{\circ} \mathrm{C}$ and expression of DF-subcomplex was induced by the addition of $2 \mathrm{mM}$ isopropyl $\beta$-D-thiogalactopyranoside. Cells were harvested 20 hours after induction by centrifugation at $6,000 \mathrm{rpm}$ for 10 minutes. The cells were suspended in $20 \mathrm{~mL}$ of buffer $\mathrm{J}$ ( $20 \mathrm{mM}$ potassium $\mathrm{P}_{\mathrm{i}}(\mathrm{pH} 8.0), 300 \mathrm{mM} \mathrm{NaCl}$ and $20 \mathrm{mM}$ Imidazole) supplemented with $100 \mu \mathrm{L}$ of $100 \mathrm{mM}$ PMSF solution, and then disrupted by sonication. After removal of cell debris by centrifugation at $10,000 \mathrm{rpm}$ for 20 minutes at $4{ }^{\circ} \mathrm{C}$, the solution was filtered and applied to a Ni-NTA column. After washing with buffer J, DF-subcomplex was eluted with buffer $\mathrm{K}\left(20 \mathrm{mM}\right.$ potassium $\mathrm{P}_{\mathrm{i}}(\mathrm{pH} 8.0), 300$ $\mathrm{mM} \mathrm{NaCl}$ and $500 \mathrm{mM}$ Imidazole). The eluted sample and TEV protease were mixed in 10:1 molar ratio and dialyzed against buffer $\mathrm{L}\left(20 \mathrm{mM}\right.$ potassium $\mathrm{P}_{\mathrm{i}}(\mathrm{pH} 8.0), 50 \mathrm{mM} \mathrm{NaCl}$, and $\left.1 \mathrm{mM} \mathrm{DTT}\right)$ overnight. The dialyzed sample was spun down at $10,000 \mathrm{rpm}$ for 20 minutes at $4{ }^{\circ} \mathrm{C}$, and then applied to PD10 column for changing the buffer to buffer J. The eluted sample was applied to a Ni-NTA column and the flow thorough was collected. After adding $1 \mathrm{mM}$ DTT, the sample was concentrated with a Vivaspin20 5,000 MWCO (Sartorius) and then passed through a Superdex 75 column (GE Helthcare) 
equilibrated with buffer $\mathrm{M}(20 \mathrm{mM}$ Tris- $\mathrm{HCl}(\mathrm{pH} 8.0), 150 \mathrm{mM} \mathrm{NaCl})$. The purified proteins were 


\section{Expression and purification of the $\mathrm{A}_{3}(\mathrm{De})_{3}$ for crystallization} sample and TEV protease were mixed in 10:1 molar ratio and dialyzed against buffer O (20 mM Tris$\mathrm{HCl}(\mathrm{pH} 8.0)$ and $50 \mathrm{mM} \mathrm{NaCl}$ ). This dialyzed samples were applied to a Ni-NTA column and the flow through was collected. Then, the sample was loaded onto a HiTrap Q HP column (GE Healthcare Life Sciences) equilibrated with buffer $\mathrm{O}$, and then eluted with a linear gradient of buffer $\mathrm{O}$ with 50-1,000 $\mathrm{mM} \mathrm{NaCl}$ in $20 \mathrm{~min}$ at flow rate of $1.0 \mathrm{ml} \mathrm{min}^{-1}$. The concentrated sample with a Vivaspin20 5,000 


\section{Preparation of the design mutants}

Technologies). The purification and expression were carried out with the same method as the original

design. The DNA sequence were confirmed by DNA sequencing analysis (Fasmac).

\section{Circular Dichroism measurement}

Thermal denaturation experiments for the designed B-subunit and the wild-type B-subunit were carried out by using the Circular Dichroism spectrometer, J-1500KS (JASCO). Far-ultraviolet circular

dichroism spectra at $220 \mathrm{~nm}$ along the increase of temperature in steps of $1.0{ }^{\circ} \mathrm{C} / \mathrm{min}$ with $60 \mathrm{~s}$ of equilibration time were collected for $5 \mu \mathrm{M}$ protein samples in buffer $\mathrm{F}$ in a 1-cm-path-length cuvette. 


\section{Fluorescence polarization measurement for evaluating nucleotide-binding affinity}

\section{of the $A_{3}(D e)_{3}$ complex}

Fluorescence polarization-based affinity measurements for the wild-type $\mathrm{A}_{3} \mathrm{~B}_{3}$ complex and the designed $\mathrm{A}_{3}(\mathrm{De})_{3}$ complex were performed using the fluorescent-labeled nucleotides, Mant-ADP and Mant-AppNHp (Jena Bioscience), in $100 \mathrm{nM}$. The changes in fluorescence anisotropy (r) of the fluorescent-labeled nucleotides mixed with the protein samples in Greiner black flat bottom 96 well plates, against the increase of the protein concentrations, were observed after 1 hour equilibration at room temperature on a Spark 10M (TECAN) using $360 \mathrm{~nm}$ excitation and $465 \mathrm{~nm}$ emission filters with $35 \mathrm{~nm}$ bandwidth filters. Buffer D was used for all measurement. Equilibrium dissociation constants $\left(K_{\mathrm{d}}\right)$ were determined by the fitting to eq 1 with the anisotropy plots averaged over period of 10 min (20 measurements), where $A$ is the experimentally measured anisotropy, $A_{f}$ is anisotropy of the free ligand, $A_{b}$ is the anisotropy of the fully bound ligand, $[\mathrm{L}]_{\mathrm{T}}$ is the total ligand concentration, and $[\mathrm{R}]_{\mathrm{T}}$ is the total protein concentration. The $K_{\mathrm{d}}$ values were determined by averaging the values from three independent measurements.

$$
A=A_{f}+\left(A_{b}-A_{f}\right) *\left(\frac{\left([L]_{T}+K_{D}+[R]_{T}\right)-\sqrt{\left(-[L]_{T}-K_{D}-[R]_{T}\right)^{2}-4[L]_{T}[R]_{T}}}{2[L]_{T}}\right) \quad \text { eq } 1
$$

Note that the measured nucleotide affinities of the wild-type $\mathrm{A}_{3} \mathrm{~B}_{3}$ complex are possibly underestimated by the binding at the second catalytic site, since the $100 \mathrm{nM}$ nucleotide concentration, 
bioRxiv preprint doi: https://doi.org/10.1101/2020.09.09.288571; this version posted September 9, 2020. The copyright holder for this preprint (which was not certified by peer review) is the author/funder. All rights reserved. No reuse allowed without permission.

422 which is required to detect the fluorescence polarization of Mant, is not low enough against the binding

423 affinity. 


\section{Reconstitution experiments of the $A_{3}(D e)_{3} D F$ complex from the $A_{3}(D e)_{3}$ complex}

\section{and the DF-subcomplex}

The $A_{3}(\mathrm{De})_{3} \mathrm{DF}$ complex was reconstituted from the $\mathrm{A}_{3}(\mathrm{De})_{3}$ complex and the DF-subcomplex. The purified $\mathrm{A}_{3}(\mathrm{De})_{3}$ and DF were mixed in a 1:5 molar ratio with the addition of MES-NaOH (pH6.0, 100 $\mathrm{mM}$ final concentration) and incubated for 2 hour at room temperature in the presence or absence of $20 \mu \mathrm{M}$ AMP-PNP or ADP. The samples were filtered and passed through a Superdex 200 Increase column (GE Helthcare) equilibrated with buffer Q (20 mM MES-NaOH (pH 6.5), 10\% Glycerol, 100 $\mathrm{mM} \mathrm{NaCl}, 5 \mathrm{mM} \mathrm{MgSO}_{4}$ and $2 \mathrm{mM}$ DTT). The reconstitution rate shown in Supplementary Fig. 11 was evaluated by SDS-PAGE for $1.5 \mu \mathrm{M}, 14 \mu \mathrm{L}$ of purified samples mixed with Tris-Glycine SDS buffer, and then quantified by Image ${ }^{41}$ using eq 2 , where $A_{W T}, A_{\text {Design }}$ are the optical densities of Asubunit in the wild-type complex or in the design complex, respectively, and $\mathrm{D}_{\mathrm{WT}}$ and $\mathrm{D}_{\text {Design }}$ are those of D-subunit.

$$
\left(\mathrm{A}_{\mathrm{WT}} / \mathrm{A}_{\text {Design }}\right) \times \mathrm{D}_{\text {Design }} / \mathrm{D}_{\mathrm{WT}}
$$

eq 2 


\section{Crystallization, data collection and structure determination}

The crystals were appeared in 1-2 weeks at $293 \mathrm{~K}$. The crystals were soaked in cryo-protectant solutions with an increasing concentration of $10 \%(\mathrm{v} / \mathrm{v})$ glycerol. For $\mathrm{A}_{3}(\mathrm{De})_{3} \_(\mathrm{ANP})_{1 \text { cat }}$, $\mathrm{A}_{3}(\mathrm{De})_{3}$ empty crystals were soaked for 5 hours in $20 \mu \mathrm{M}$ AMP-PNP, $200 \mu \mathrm{M} \mathrm{MgSO}_{4}$ and $10 \%$ glycerol. For $\mathrm{A}_{3}(\mathrm{De})_{3} \_(\mathrm{ADP} \cdot \mathrm{Pi})_{1 \text { cat }}(\mathrm{ADP})_{2 \text { cat,2non-cat, }} \mathrm{A}_{3}(\mathrm{De})_{3}$ empty crystals were soaked to ADP,

$\mathrm{MgCl}_{2}$ and glycerol for 5 hours by gradually increasing the concentration to $10 \mathrm{mM}, 10 \mathrm{mM}$ and $10 \%$,

respectively. For $A_{3}(D e)_{3} \_(A D P)_{3 c a t, 1 \text { non-cat }}$ and $A_{3}(D e)_{3} \_(A D P)_{3 c a t, 2 n o n-c a t}, A_{3}(D e)_{3}$ empty crystals were soaked to $\mathrm{ADP}, \mathrm{MgCl}_{2}$ and glycerol overnight by gradually increasing the concentration to $5 \mathrm{mM}, 5$ $\mathrm{mM}$ and $10 \%$, respectively. nitrogen. All X-ray diffraction data were collected at the wavelength $1.1 \AA$ on beamline BL-1A at 
from Enterococcus hirae (PDB 3VR2) and obtained $\mathrm{A}_{3}(\mathrm{De})_{3}$ empty structure as a search model, and manually corrected with $\mathrm{COOT}^{46}$. Figures are prepared by $\mathrm{PyMOL}^{47}, \mathrm{CueMol}^{48}$ and $\mathrm{Chimera}^{49}$.

The crystallographic and refinement statistics are summarized in Supplementary Table 9. 


\section{Single-molecule experiments of the designed V1-ATPase}

The protein sample was prepared by mixing the purified $\mathrm{A}_{3}(\mathrm{De})_{3}$ and the biotinylated DF-subcomplex

in a 1:5 molar ratio with the addition of MES-NaOH (pH6.0, 100mM final concentration), followed

by the incubation in the presence of $200 \mu \mathrm{M}$ ADP for 2 hours at room temperature. The sample was

filtered and passed through a Superdex 200 Increase column (GE Helthcare) equilibrated with buffer

$\mathrm{Q}$ and were concentrated to few $\mu \mathrm{M}$ with a Vivaspin500 5,000 MWCO. The samples were stored at $80{ }^{\circ} \mathrm{C}$.

Single-molecule experiments were carried out by the method reported in the paper ${ }^{28}$. The flow cell was prepared by covering an untreated coverglass $\left(18 \times 18 \mathrm{~mm}^{2}\right.$, Matsunami Glass $)$ on a coverglass $\left(24 \times 32 \mathrm{~mm}^{2}\right.$, Matsunami Glass $)$ treated by overnight immersion in piranha solution $\left(\mathrm{H}_{2} \mathrm{SO}_{4} / \mathrm{H}_{2} \mathrm{O}_{2}=\right.$ 3:1). After capturing the protein sample on the treated coverglass by His-tag, the streptavidin-coated 40-nm gold nanoparticle was attached to the biotinylated DF. The rotation of gold nanoparticle was observed by using an objective-type total internal reflection dark-field microscope ${ }^{50}$ constructed on an inverted microscope (IX-70, Olympus). The gold nanoparticles were illuminated by the evanescent field with the penetration depth of $100 \mathrm{~nm}$ from the glass surface. The scattered image of a rotating gold nanoparticle was recorded as a movie with a high-speed CMOS camera (FASTCAM 1024PCI, Photron) at 10,000 frames per second (fps) for almost all samples and at 27,000 fps for dwell time 
analyses of the double mutant at $3 \mathrm{mM}$ ATP. During observation and recording under the microscope,

477 ATP-regeneration system, in which ADP is rapidly regenerated to ATP by the coupling with

478 dephosphorylation of phosphoenolpyruvate catalyzed by pyruvate kinase, was used to keep ATP

479 concentration constant. 


\section{References}

1. Gavin, A.-C. et al. Proteome survey reveals modularity of the yeast cell machinery. Nature 440, 631-636 (2006).

2. Alberts, B. The Cell as a Collection of Protein Machines: Preparing the Next Generation of Molecular Biologists. Cell 92, 291-294 (1998).

3. Kuriyan, J. \& Eisenberg, D. The origin of protein interactions and allostery in colocalization. Nature 450, 983-990 (2007).

4. Makhlynets, O.V., Raymond, E.A. \& Korendovych, I.V. Design of Allosterically Regulated Protein Catalysts. Biochemistry 54, 1444-1456 (2015).

5. Liu, H. et al. Control of a biomolecular motor-powered nanodevice with an engineered chemical switch. Nature Materials 1, 173-177 (2002).

6. Lee, J. et al. Surface Sites for Engineering Allosteric Control in Proteins. Science 322, 438 (2008).

7. Feng, J. et al. A general strategy to construct small molecule biosensors in eukaryotes. eLife 4, e10606 (2015).

8. Nakamura, M. et al. Remote control of myosin and kinesin motors using light-activated gearshifting. Nature Nanotechnology 9, 693 (2014).

9. Cosentino, C. et al. Engineering of a light-gated potassium channel. Science 348, 707 (2015).

10. Zeqiraj, E. \& van Aalten, D.M. Pseudokinases-remnants of evolution or key allosteric regulators? Curr Opin Struct Biol 20, 772-81 (2010).

11. Ribeiro, A.J.M. et al. Emerging concepts in pseudoenzyme classification, evolution, and signaling. Science Signaling 12, eaat9797 (2019).

12. Manning, G., Whyte, D.B., Martinez, R., Hunter, T. \& Sudarsanam, S. The Protein Kinase Complement of the Human Genome. Science 298, 1912 (2002).

13. Zeqiraj, E., Filippi, B.M., Deak, M., Alessi, D.R. \& van Aalten, D.M.F. Structure of the LKB1STRAD-MO25 Complex Reveals an Allosteric Mechanism of Kinase Activation. Science 326, 1707 (2009).

14. Rajakulendran, T. \& Sicheri, F. Allosteric Protein Kinase Regulation by Pseudokinases: Insights from STRAD. Science Signaling 3, pe8 (2010).

15. Marshansky, V. \& Futai, M. The V-type H+-ATPase in vesicular trafficking: targeting, regulation and function. Curr Opin Cell Biol 20, 415-26 (2008).

16. Arai, S. et al. Rotation mechanism of Enterococcus hirae V1-ATPase based on asymmetric crystal structures. Nature 493, 703-7 (2013).

17. Arai, S. et al. Reconstitution in vitro of the catalytic portion (NtpA3-B3-D-G complex) of 
Enterococcus hirae V-type Na+-ATPase. Biochem Biophys Res Commun 390, 698-702 (2009).

18. Liu, Q., Kane, P.M., Newman, P.R. \& Forgac, M. Site-directed mutagenesis of the yeast VATPase B subunit (Vma2p). J Biol Chem 271, 2018-22 (1996).

19. Vasilyeva, E., Liu, Q., MacLeod, K.J., Baleja, J.D. \& Forgac, M. Cysteine scanning mutagenesis of the noncatalytic nucleotide binding site of the yeast V-ATPase. J Biol Chem 275, 255-60 (2000).

20. Mulkidjanian, A.Y., Makarova, K.S., Galperin, M.Y. \& Koonin, E.V. Inventing the dynamo machine: the evolution of the F-type and V-type ATPases. Nat Rev Microbiol 5, 892-9 (2007).

21. Matsui, T. et al. Catalytic activity of the $\alpha 3 \beta 3 \gamma$ complex of F1-ATPase without noncatalytic nucleotide binding site. J Biol Chem 272, 8215-21 (1997).

22. Walker, J.E. ATP Synthesis by Rotary Catalysis (Nobel lecture). Angew Chem Int Ed Engl 37, 2308-2319 (1998).

23. Walker, J.E., Saraste, M., Runswick, M.J. \& Gay, N.J. Distantly related sequences in the alphaand beta-subunits of ATP synthase, myosin, kinases and other ATP-requiring enzymes and a common nucleotide binding fold. The EMBO journal 1, 945-951 (1982).

24. Saraste, M., Sibbald, P.R. \& Wittinghofer, A. The P-loop--a common motif in ATP- and GTPbinding proteins. Trends Biochem Sci 15, 430-4 (1990).

25. Tinberg, C.E. et al. Computational design of ligand-binding proteins with high affinity and selectivity. Nature 501, 212-216 (2013).

26. Dou, J. et al. De novo design of a fluorescence-activating beta-barrel. Nature 561, 485-491 (2018).

27. Bick, M.J. et al. Computational design of environmental sensors for the potent opioid fentanyl. eLife 6, e28909 (2017).

28. Iida, T. et al. Single-molecule analysis reveals rotational substeps and chemo-mechanical coupling scheme of Enterococcus hirae V1-ATPase. J Biol Chem 294, 17017-17030 (2019).

29. Imamura, H., Funamoto, S., Yoshida, M. \& Yokoyama, K. Reconstitution in vitro of V1 complex of Thermus thermophilus V-ATPase revealed that ATP binding to the A subunit is crucial for V1 formation. J Biol Chem 281, 38582-91 (2006).

30. Greenfield, N.J. Using circular dichroism collected as a function of temperature to determine the thermodynamics of protein unfolding and binding interactions. Nat Protoc 1, 2527-35 (2006).

31. Parsonage, D., Al-Shawi, M.K. \& Senior, A.E. Directed mutations of the strongly conserved lysine 155 in the catalytic nucleotide-binding domain of beta-subunit of F1-ATPase from Escherichia coli. J Biol Chem 263, 4740-4 (1988).

32. Maruyama, S. et al. Metastable asymmetrical structure of a shaftless V1 motor. Science 
Advances 5, eaau8149 (2019).

33. Kon, T. et al. The 2.8 A crystal structure of the dynein motor domain. Nature 484, 345-50 (2012).

34. Allingham, J.S., Sproul, L.R., Rayment, I. \& Gilbert, S.P. Vik1 Modulates Microtubule-Kar3 Interactions through a Motor Domain that Lacks an Active Site. Cell 128, 1161-1172 (2007).

35. Stadtmueller, B.M. \& Hill, C.P. Proteasome Activators. Molecular Cell 41, 8-19 (2011).

36. Wang, J. et al. Ligand-triggered allosteric ADP release primes a plant NLR complex. Science 364, eaav5868 (2019).

37. Leaver-Fay, A. et al. ROSETTA3: an object-oriented software suite for the simulation and design of macromolecules. Methods Enzymol 487, 545-74 (2011).

38. Kothiwale, S., Mendenhall, J.L. \& Meiler, J. BCL::Conf: small molecule conformational sampling using a knowledge based rotamer library. J Cheminform 7, 47 (2015).

39. D.A. Case, V.B., J.T. Berryman, R.M. Betz, Q. Cai, D.S. Cerutti, T.E. Cheatham, III, T.A. Darden, R.E. Duke, H. Gohlke, A.W. Goetz, S. Gusarov, N. Homeyer, P. Janowski, J. Kaus, I. Kolossváry, A. Kovalenko, T.S. Lee, S. LeGrand, T. Luchko, R. Luo, B. Madej, K.M. Merz, F. Paesani, D.R. Roe, A. Roitberg, C. Sagui, R. Salomon-Ferrer, G. Seabra, C.L. Simmerling, W. Smith, J. Swails, R.C. Walker, J. Wang, R.M. Wolf, X. Wu and P.A. Kollman. AMBER 14. (University of California, San Francisco., 2014).

40. Meagher, K.L., Redman, L.T. \& Carlson, H.A. Development of polyphosphate parameters for use with the AMBER force field. Journal of Computational Chemistry 24, 1016-1025 (2003).

41. Schneider, C.A., Rasband, W.S. \& Eliceiri, K.W. NIH Image to ImageJ: 25 years of image analysis. Nature Methods 9, 671-675 (2012).

42. Kabsch, W. XDS. Acta Crystallographica Section D 66, 125-132 (2010).

43. McCoy, A.J. et al. Phaser crystallographic software. Journal of Applied Crystallography 40, 658-674 (2007).

44. Adams, P.D. et al. PHENIX: a comprehensive Python-based system for macromolecular structure solution. Acta Crystallographica Section D 66, 213-221 (2010).

45. Murshudov, G.N., Vagin, A.A. \& Dodson, E.J. Refinement of Macromolecular Structures by the Maximum-Likelihood Method. Acta Crystallographica Section D 53, 240-255 (1997).

46. Emsley, P., Lohkamp, B., Scott, W.G. \& Cowtan, K. Features and development of Coot. Acta Crystallographica Section D 66, 486-501 (2010).

47. Schrodinger, LLC. The PyMOL Molecular Graphics System, Version 2.1. (2018).

48. CueMol: Molecular Visualization Framework (http://www.cuemol.org/).

49. Pettersen, E.F. et al. UCSF Chimera-A visualization system for exploratory research and analysis. Journal of Computational Chemistry 25, 1605-1612 (2004). 
584 50. Ueno, H. et al. Simple Dark-Field Microscopy with Nanometer Spatial Precision and Microsecond Temporal Resolution. Biophysical Journal 98, 2014-2023 (2010). 


\section{Acknowledgement}

We thank Prof. T. Murata at Chiba University for helpful suggestions; Dr. M. Kondo at ExCELLS

and Dr. F. Kawai at Yamagata University for discussion about protein expression and purification; Dr.

590

591

592

593

H. Ueno and Dr. Y. Minagawa at the University of Tokyo for advice about single-molecule

experiments; Prof. T. Kinoshita at Osaka Prefecture University for discussion about pseudo-kinases;

Prof. S. Akiyama at the Institute for Molecular Science and Prof. S. Takada at Kyoto University for comments on the manuscript; and Dr. R. Koga at ExCELLS for general discussion. We thank the Research Center for Computational Science (RCCS), Okazaki, Japan for providing its computational resources; the staff at the Photon Factory (PF) at KEK for assistance in synchrotron experiments and data collection for crystallographic analyses under the approval of PF program advisory committee (Proposal No. 2017G141); and for the support of the Basis for Supporting Innovative Drug Discovery and Life Science Research (BINDS) from AMED under Grant Number JP20am0101071 (supporting number BINDS0471). This work was supported by a Grant-in-Aid for Scientific Research on Innovative Areas "Molecular Engine" (JSPS KAKENHI Grant Number $18 H 05420$ to T. K. and N. K. and $18 \mathrm{H} 05424$ to R. I.), by the National Institute for Natural Sciences (NINS), Okazaki Institute for Integrative Bioscience (Orion Project Grant Number 10341630611 to T.K. N. K. and R. I.), and by the Japan Science and Technology Agency (JST) Precursory Research for Embryonic Science and 


\section{Author Contributions}

T. K., R. I. and N. K. designed the research; T. K. computationally designed the ATP binding site; T.

K. expressed and purified protein samples; T. K. performed biochemical measurements; T. K. and M.

T. performed crystallography experiments and analyzed the data; T. K. and T. I. performed single-

\section{${ }_{613}$ Competing financial interests}

\section{Additional Information}


bioRxiv preprint doi: https://doi.org/10.1101/2020.09.09.288571; this version posted September 9, 2020. The copyright holder for this preprint (which was not certified by peer review) is the author/funder. All rights reserved. No reuse allowed without permission.

a

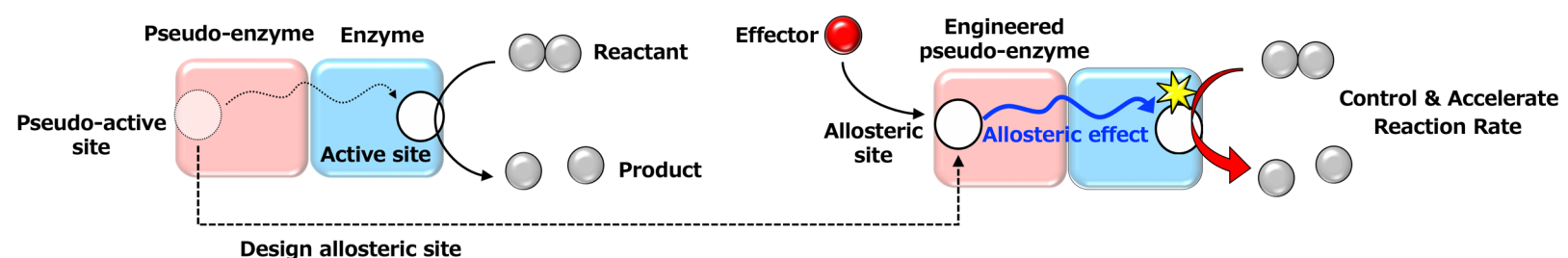

b

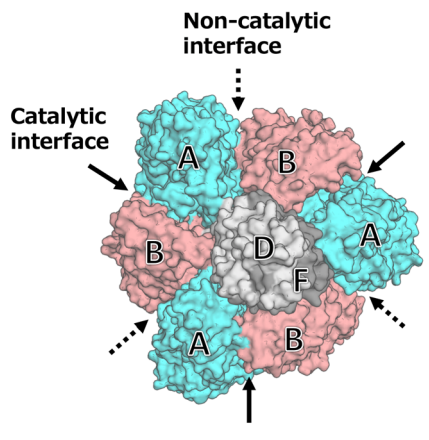

Enterococcus hirae $\mathrm{V}_{1}$

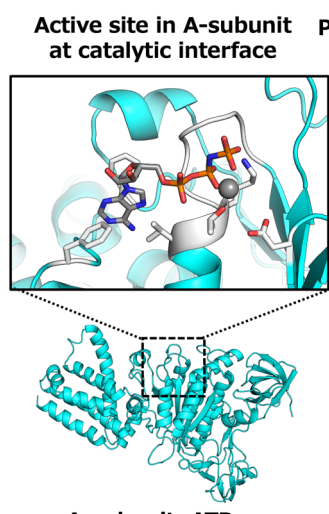

A-subunit: ATPase
Pseudo-active site in B-subunit at non-catalytic interface
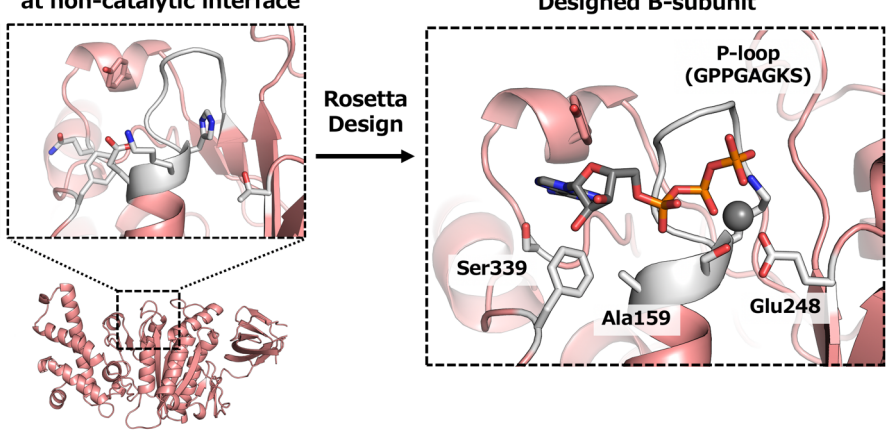

B-subunit: Pseudo-enzyme 
Fig. 1. De Novo Design of an allosteric (ATP binding) site at the pseudo-active site of $\mathrm{V}_{1}$-ATPase.

a, Strategy to de novo design of allosteric control into protein complexes by engineering the pseudoactive site. b, Overview of design scheme. Left: Catalytic and non-catalytic interfaces, indicated by solid and dotted arrows respectively, in the hexameric ring of $\mathrm{V}_{1}$ consisting of A-subunits (cyan) and their pseudo-enzyme B-subunits (salmon pink). The rotor of the D- and F- subunits (gray) is located in the center of the ring. Middle: The structures of A- and B-subunits with the active and pseudo-active sites, respectively. Right: An ATP binding site created at the pseudo-active site using the protein design software Rosetta; gray color residues were selected for the design (11 residue positions). The residues changed from the original sequence by the design are denoted with characters: the P-loop for binding to the phosphate group of ATP was built at the residue positions 151-158 with the amino acid sequence, GPPGAGKS; the Walker-B motif coordinating magnesium ion was built with glutamic acid at the residue position 248; the nucleotide-binding pocket was made with the alanine at 159 , creating space for the binding of the sugar group of ATP (originally glutamic acid), and serine 339, making a hydrogen bond with the adenine ring. 
a

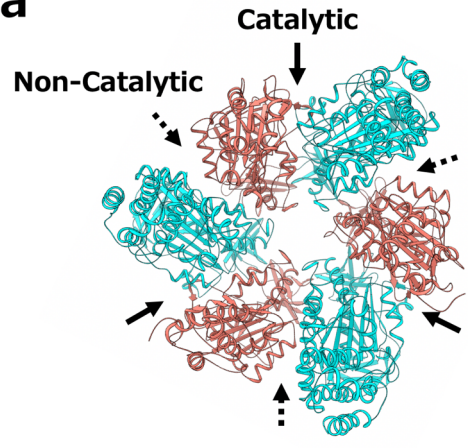

$A_{3}(D e)_{3}$ empty b

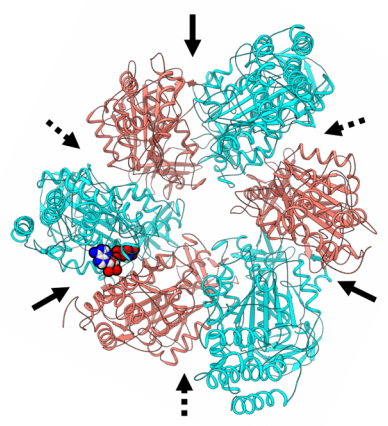

$A_{3}(\text { De })_{3}(\text { ANP })_{1 \text { cat }}$ PDB: 7COQ
C

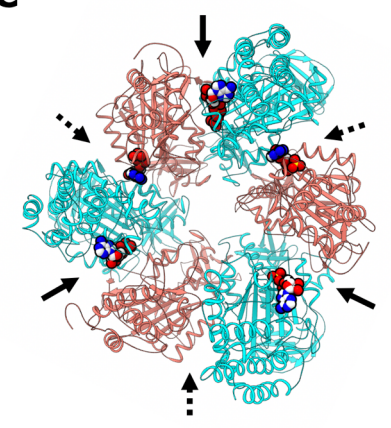

$A_{3}(D e)_{3}(A D P \cdot P i)_{1 \text { cat }}(A D P)_{2 \text { cat,2non-cat }}$ PDB: 7COR d

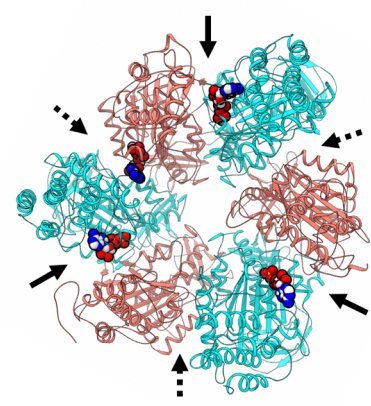

$A_{3}(D e)_{3}(A D P)_{3 c a t, 1 \text { non-cat }}$ PDB: 7COS e

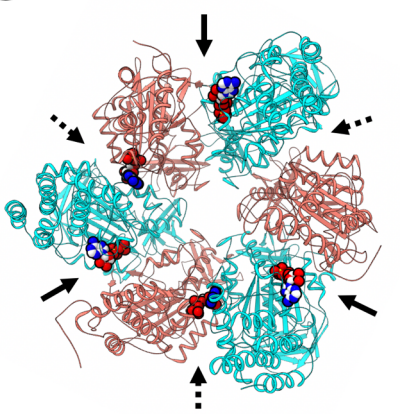

$A_{3}(D e)_{3}(A D P)_{3 c a t, 2 n o n-c a t}$ PDB: 7COS 
Fig. 2. Solved five crystal structures of the $A_{3}(\mathrm{De})_{3}$ complex in various conditions. structure bound to 3 ADPs in the catalytic interfaces and 2 ADPs in the designed non-catalytic interfaces $\left(2.9 \AA\right.$ resolution): $\mathrm{A}_{3}(\mathrm{De})_{3} \_(\mathrm{ADP} \cdot \mathrm{Pi})_{1 \text { cat }}(\mathrm{ADP})_{2 \text { cat,2non-cat. }}(\mathrm{D}) \mathrm{A}_{3}(\mathrm{De})_{3}$ complex structure 641 bound to 3 ADPs in the catalytic interfaces and an ADP in a designed non-catalytic interface (3.95 642 resolution): $\mathrm{A}_{3}(\mathrm{De})_{3}(\mathrm{ADP})_{3 \text { cat, } 1 \text { non-cat. }}(\mathrm{E}) \mathrm{A}_{3}(\mathrm{De})_{3}$ complex structure bound to 3 ADPs in the catalytic 643 interfaces and 2 ADPs in the designed non-catalytic interfaces $(3.95 \AA$ resolution): 
a

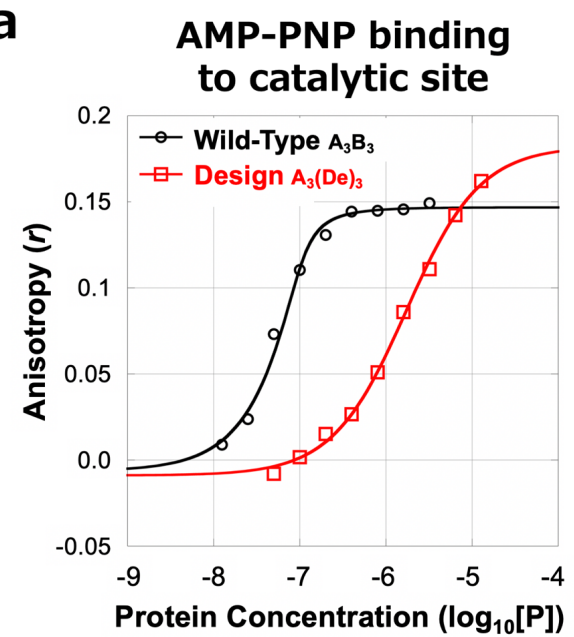

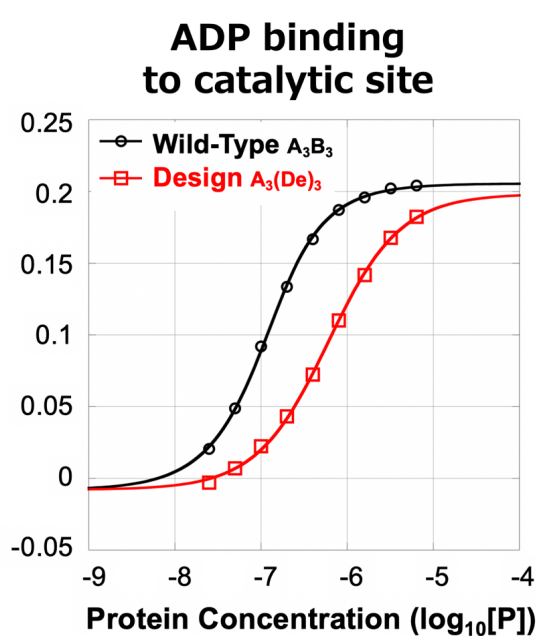

Design $\mathrm{A}_{3}(\mathrm{De})_{3} \mathrm{empty}_{\mathrm{B}}$

b

Wild-type $A_{3} B_{3} \_$empty

\section{A-subunits}

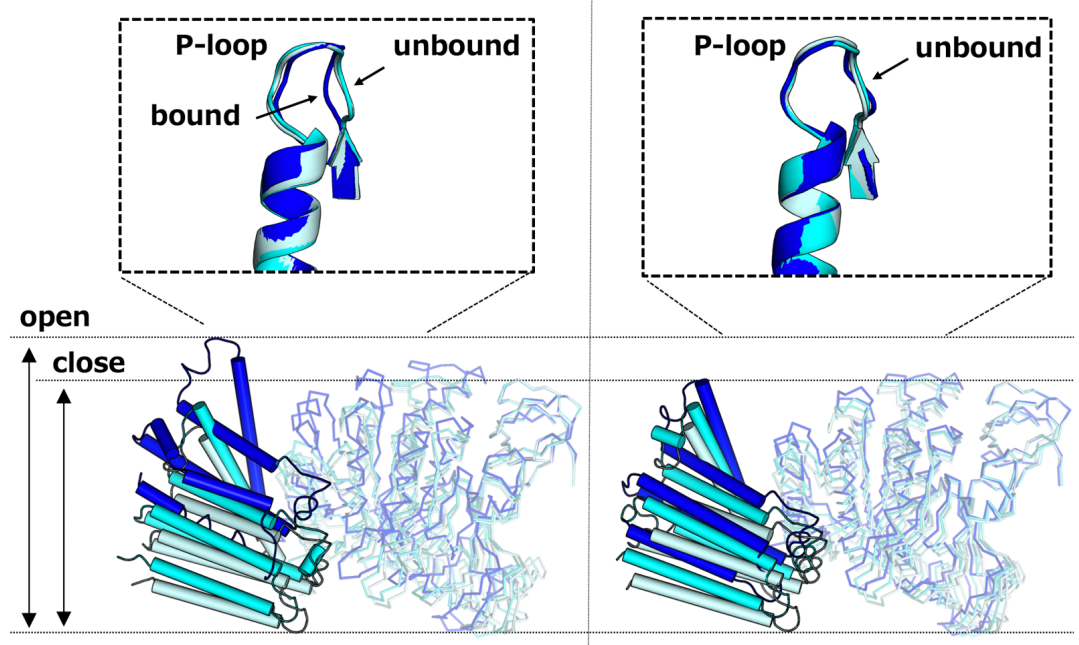

C Hexametric ring complex
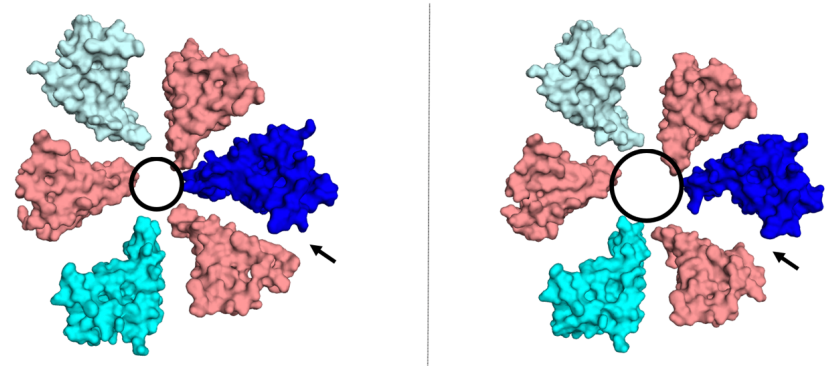

d

\section{B-subunits}

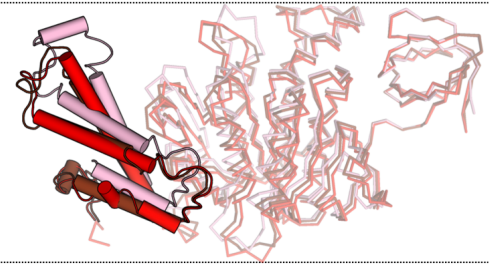


Fig. 3. Creation of ATP binding sites in the B-subunit induced conformational changes of the A-

a, The nucleotide binding affinities of the wild-type $A_{3} B_{3}$ and $A_{3}(D e)_{3}$ complexes to AMP-PNP (top) A-subunit structures in the wild-type $\mathrm{A}_{3} \mathrm{~B}_{3}$ (PDB: 3VR2) (left) and $\mathrm{A}_{3}(\mathrm{De})_{3}$ empty (PDB: 7COP)

651 (right), using the $\beta$-barrel domains (residues 1-71), together with the close-up views for the structures 652 around the P-loop. c, Ring complex conformations of the wild-type $\mathrm{A}_{3} \mathrm{~B}_{3}$ complex (left) and $653 \mathrm{~A}_{3}(\mathrm{De})_{3}$ empty (right). The C-terminal domains of $\mathrm{A}$ - and $\mathrm{B}$ - subunits viewed from the N-terminal $\beta$ 654 barrel side, are shown. The circles and arrows show the central pore and the catalytic interface at which 655 the largest conformational change is observed. d, Superpositions of the three B-subunit structures. 
a
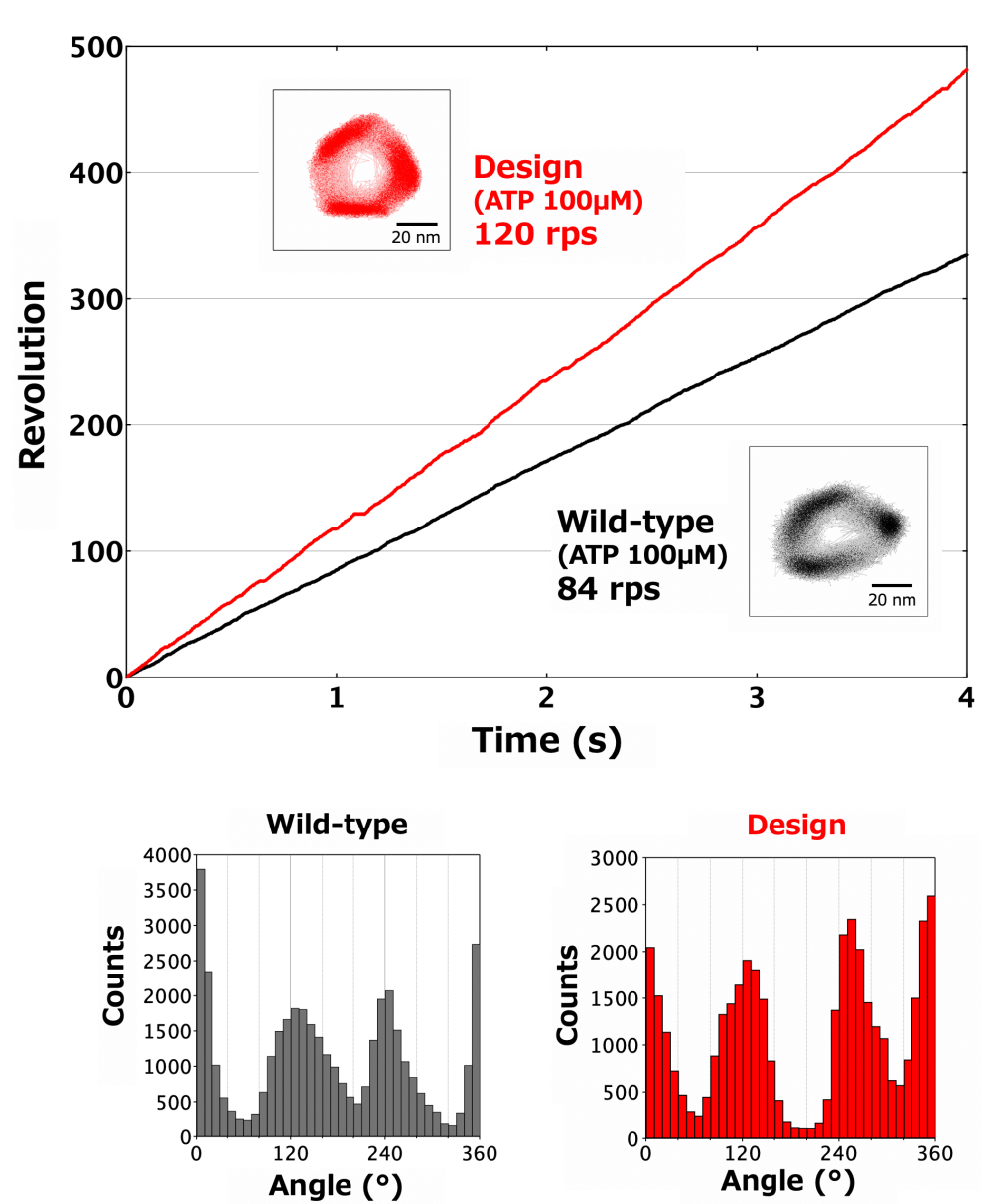

b
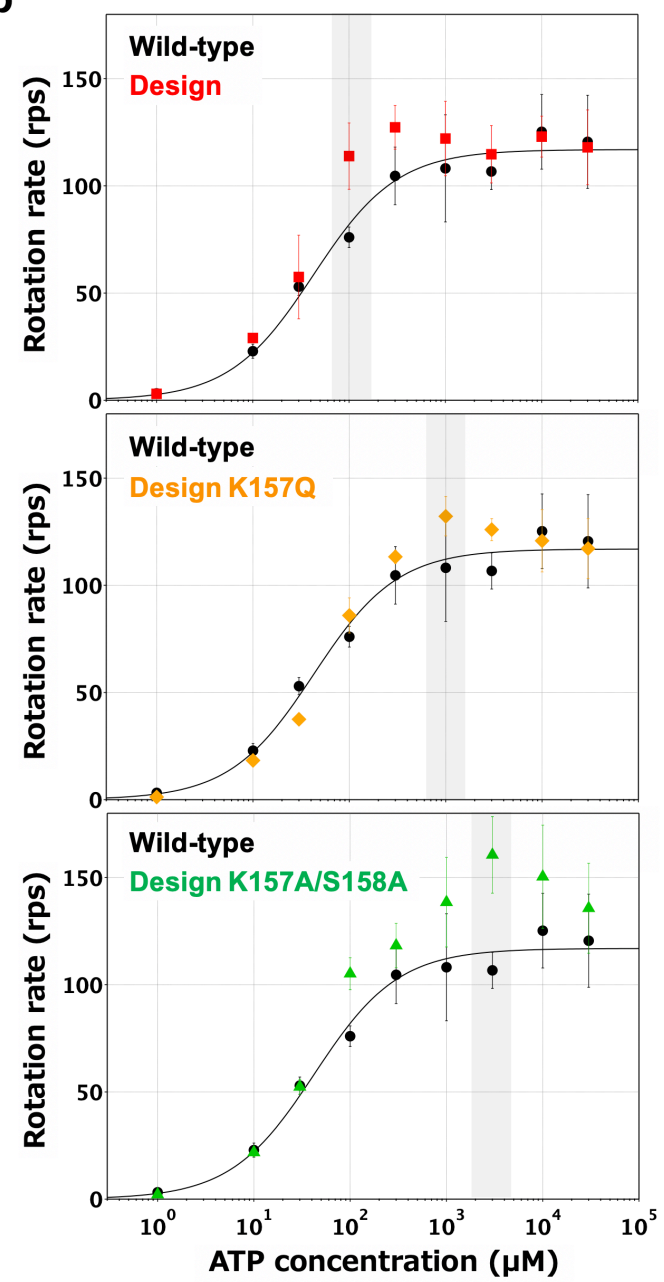
Fig. 4. ATP binding to the designed site accelerates rotation rate allosterically.

a, A typical rotation time course of the designed $V_{1}$ (red) and that of the wild-type $V_{1}$ (green) ${ }^{28}$, at 100

$\mu \mathrm{M}$ ATP. The insets show the rotation xy-trajectory. The angle distributions are shown at the bottom.

b, [ATP] dependence of rotational rates for the wild-type (black) ${ }^{28}$, the designed $\mathrm{V}_{1}$ (red), the design 
a
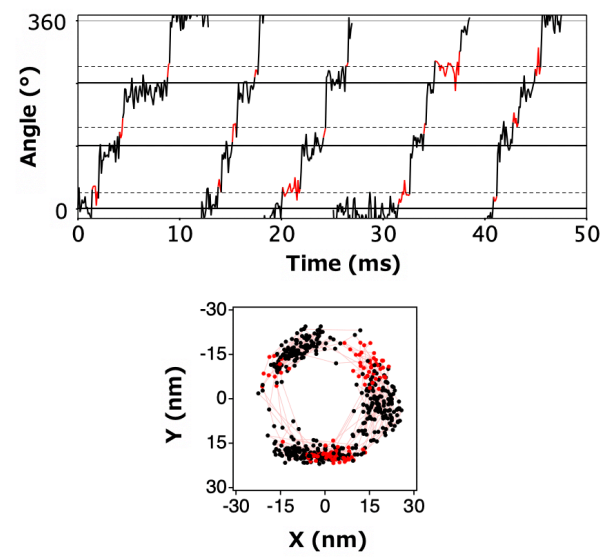

C
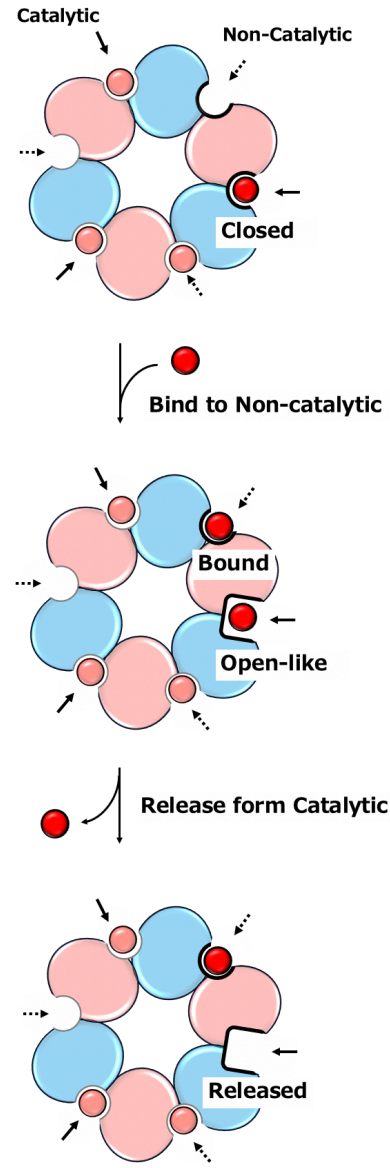

e b

Wild-type, Design, Design K157A/S158A

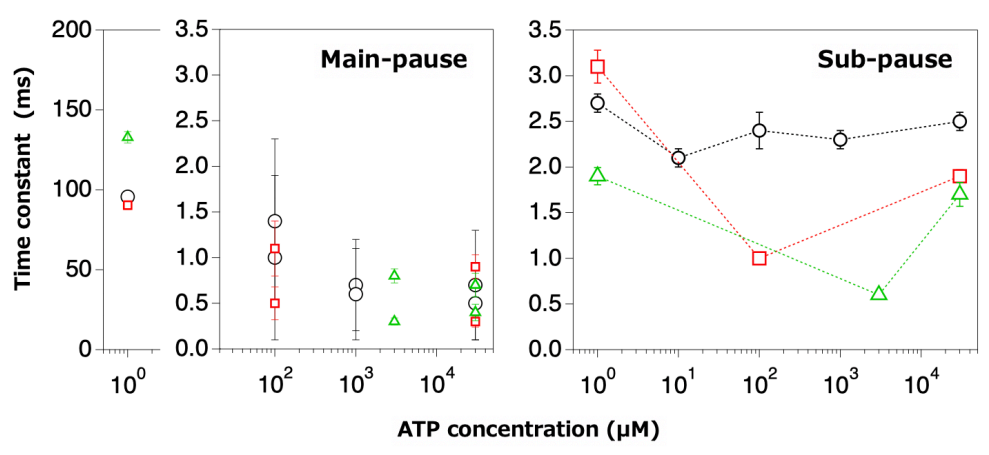

d

$A_{3}(D e)_{3}(A D P)_{3 c a t, 1 \text { non-cat }}$
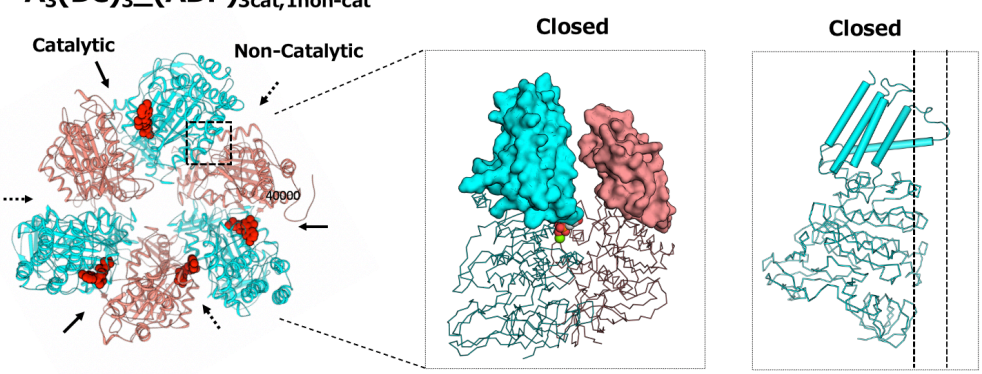

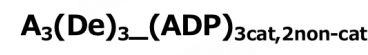
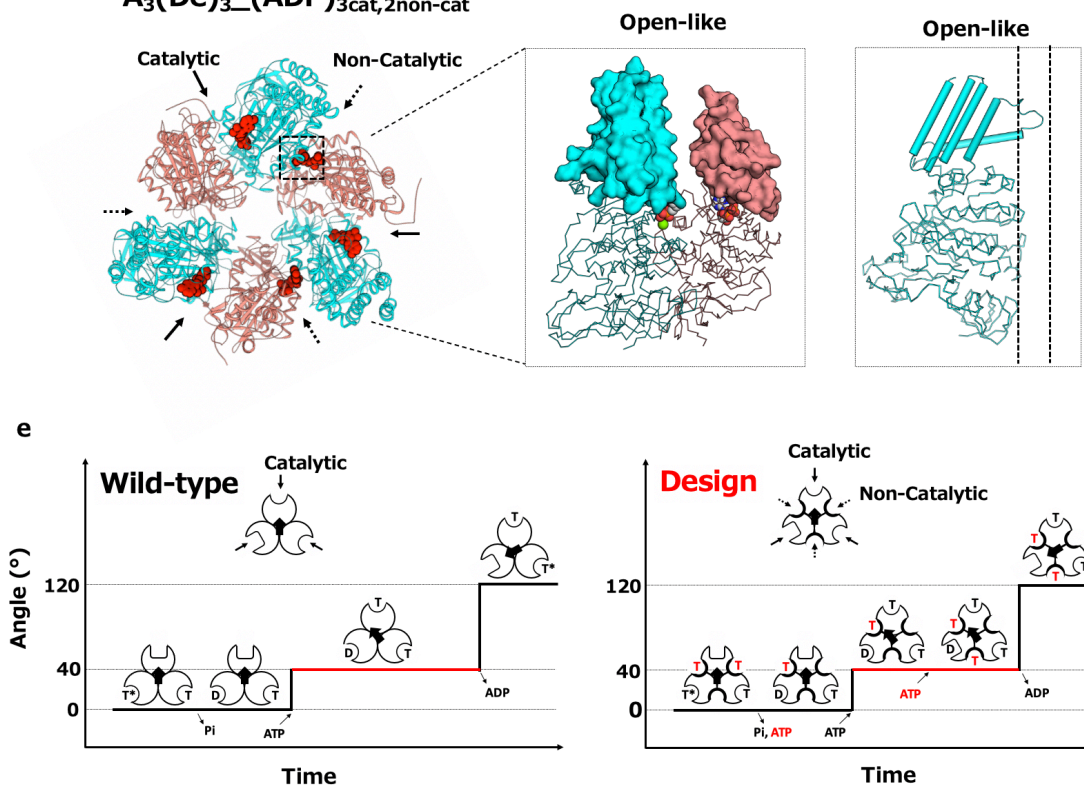
Fig. 5. The mechanism of allosteric acceleration, revealed by analysis of rotation sub-steps and

\section{solved structures.}

a, A close-up rotation time course of the design at $100 \mu \mathrm{M}$ ATP and the rotation xy-trajectory. The main-pause and sub-pause are black and red, respectively. b, Duration times at different [ATP] for the two pauses for the wild-type $\mathrm{V}_{1}$ (black), the designed $\mathrm{V}_{1}$ (red), and the design double mutant K157A/S158A (green). See Supplementary Fig. 13 for distributions of the duration time. Note that for the main pauses at 100-3000 $\mu \mathrm{M}$ ATP, two time constants were obtained for each [ATP] assuming consecutive reactions (see Supplementary Fig. 13). c, Structure-based interpretation on the ADPrelease promoted by the allosteric effect. Ellipses indicate A-subunits (cyan) and designed B-subunits (salmon). Nucleotides are red (or salmon pink) circles. d, Comparison of $\mathrm{A}_{3}(\mathrm{De})_{3}$ (ADP) $)_{3 \text { cat, } 1 \text { non-cat }}$ (top) and $\mathrm{A}_{3}(\mathrm{De})_{3}(\mathrm{ADP})_{3 \mathrm{cat}, 2 \text { non-cat }}$ (bottom). The hexameric structures viewed from the C-terminal domain of the A- and B-subunit (left) and the structures of the catalytic interfaces and the A-subunits, which form the closed and open-like conformations, respectively (middle and right). ADP molecules are shown as red spheres. e, A rotation scheme for the wild-type, proposed by Iida et al. ${ }^{28}$ (left). Proposed hypothetical rotation scheme for the design (right). ATP and ADP are represented by T and D, respectively. Red indicates nucleotides bound to non-catalytic interfaces. 